Artículo

\title{
El profesor ingenuo
}

\author{
The naïve teacher \\ Nelson Rubén Martínez Reyes* \\ nelson.martinez@udb.edu.sv
}

ISSN 1996-1642, Editorial Universidad Don Bosco, año 8, No.14, Julio-Diciembre de 2014, pp.5-18 Recibido: 1 de julio de 2014 Aprobado: 25 de julio de 2014.

\section{Resumen}

En el presente artículo el autor explora la dimensión de la ingenuidad como una condición adscrita al profesor que, en mayor o menor medida, configura no solo su visión sobre educación y los procesos formativos sino también configura y condiciona su práctica educativa. Se destaca en el artículo como el profesor ingenuo, que es una mujer u hombre crédulo, confiado, acrítico, no es capaz de dilucidar las intenciones, las consecuencias, las incoherencias y contradicciones que plantea la educación ni de discernir el entramado socio-político-económico-cultural que subyace su práctica educativa, la escuela y el currículo porque dichas acciones suceden en forma oculta $y$ disfrazada. Dicho análisis se hace desde la pedagogía crítica.

Palabras clave: ingenuidad, inocencia, educación, profesor, escuela, currículo.

\section{Abstract}

In this paper the author examines naivety as a teacher's condition that to some extent shapes not only his or her vision about education but also shapes his or her teaching practice. The author unfolds in his argument how the naiive teacher, who is a credulous, trustful and uncritical man or a woman, is not able to fathom the intentions, consequences, inconsistencies and contradictions posed by education and fail to reveal the intricate sociopolitical-economical-cultural tangle that underlies his o her teaching practice, school and curriculum since those actions happen in hidden and disguised ways. The analysis is carried out under the critical pedagogy approach.

Keywords: naivety, innocence, education, teacher, school, curriculum.

A Alexandra.

* El autor es profesor investigador adscrito a la Escuela de Educación de la Universidad Don Bosco y es también candidato a doctor en educación de la Universidad de Costa Rica. 


\section{Introducción}

Los profesores son a menudo etiquetados y clasificados en diversas categorías. Dichas categorizaciones, unas más sofisticadas y formales que otras, pueden provenir de los estudiantes mismos basándose en su propia experiencia, de las instituciones educativas donde estos laboran, de los ministerios de educación y sistemas educativos o de los tratados de pedagogía y didáctica. Los criterios pueden ser también de diversa índole: el pensamiento del profesor, personalidad o cualidad personal preponderante, la práctica pedagógica que denota una forma particular de enseñar, la burocracia institucional $\mathrm{O}$ incluso otros parámetros menos ortodoxos. Así, atendiendo a esa variedad de posibilidades, se tienen profesores que se clasifican como autoritarios y dictadores o democráticos, comprometidos y entregados o no comprometidos, pedagógica y didácticamente competentes $\mathrm{O}$ incompetentes, efectivos y eficientes que realmente hacen que el estudiante aprenda o inefectivos y deficientes que solo pasan el tiempo, novicios o experimentados, actualizados u obsoletos, dinámicos y divertidos o desganados y aburridos, estrictos o flojos, gruñones y malhumorados o los siempre sonrientes. Las categorías pueden continuar indefinidamente.

Dentro de esa lista de opciones aparece una posible categorización: la del profesor ingenuo. Sin embargo, aquí no se busca etiquetar o categorizar al profesor, sino analizar, desde la pedagogía crítica, la "ingenuidad" como una dimensión adscrita al profesor que se traduce en una particular actitud, visión o forma de entender la educación y los procesos formativos y, por tanto, de materializar la práctica pedagógica. Tampoco se pretende aquí analizar las diversas clasificaciones del profesor, sino reflexionar en una condición en particular que no necesariamente está en contraposición con otra, pero que si así fuera, esa condición opuesta sería la del profesor crítico.

\section{La ingenuidad y el profesor}

La noción detrás de la "ingenuidad" tiene que ver con "candor" y "falta de malicia". El significado de la ingenuidad está estrechamente relacionado con la idea de "sinceridad", "sencillez" y "pureza del ánimo". Igualmente implica "inocencia", "sin dobleces" o "sin segundas intenciones". De ahí que el profesor ingenuo se presenta en mayor o menor medida con atributos, formas de sentir, pensar y actuar que se relacionan con la inocencia y la pureza de ánimo.

El profesor ingenuo es un hombre o mujer que como profesor demuestra inocencia, buena fe y cierto desconocimiento de las cosas. Es más, se manifiesta como alguien que no sólo no sabe; sino que tampoco necesita saber, lo cual no implica que sea falto de entendimiento o razón, ni necesariamente un lego o un inexperto, aunque tampoco se presenta como intelectual. Se presenta como alguien que se encuentra a salvo de pensamientos nocivos, libre de malas intenciones y con una visión romántica de la realidad. El profesor ingenuo es a menudo bien intencionado, tiene los mejores propósitos, tiene un corazón noble, una mente razonablemente limpia y un espíritu sensible. No está "contaminado" con ideas extrañas, subversivas, retorcidas ni heréticas. 
El profesor ingenuo es crédulo, confiado y acrítico. Cree en lo que enseña, tiene firme convicción de la nobleza de su profesión y proyecta con optimismo su trabajo. Cree en la educación, en la escuela, en su labor educativa y en sus muchachos. Confía en las personas y en sus buenas intenciones. No cuestiona ni se cuestiona a sí mismo, no mira consecuencias ulteriores ni intereses escondidos. Es por tanto, en muchos aspectos, un sujeto sencillo, a menudo inofensivo y en apariencia no representa peligro.

Si bien se reconoce que no hay práctica educativa neutral ni enseñanza ingenua (Freire, 1998; Apple, 1986; Giroux, 1997), dado que la enseñanza no es una actividad casual o sin consecuencias. Es decir, se asume aquí que la enseñanza escolar es una actividad intencional, estructurada y conlleva ciertos objetivos explícitos y escondidos. Sin embargo, el carácter ingenuo se sustenta en la visión particular que el profesor le imprime a su práctica y en el hecho que a menudo ésta se realiza de forma inconsciente, sin que el profesor tenga conocimiento claro de la naturaleza contradictoria de ella y de la educación, ni de sus propósitos y consecuencias ocultas.

Sin embargo, a este punto, se debe advertir que a pesar de las buenas intenciones y la buena fe del profesor ingenuo, sus resultados no siempre son los esperados, ya que a menudo las acciones humanas, como lo explican Romero y Gómez (2007), se ven sorprendidas por consecuencias inadvertidas, no buscadas ni previstas que pueden desencadenar involuntariamente la producción y reproducción de ciertas dinámicas docentes, educativas y sociales indeseadas o incluso contraproducentes. De igual manera, resulta útil retomar la advertencia de Lerner (1996) cuando dice que una cosa es lo que la escuela y el profesor enseñan y otra la que el alumno aprende.

Se reconoce también que el comportamiento, las actuaciones del profesor no son sólo el resultado de objetivación de su entorno social, sino también son la concreción de sus nociones, sentimientos e inclinaciones. Es necesario recordar que las prácticas de enseñanza son también un producto de la subjetivación de la realidad del profesor, de su "mundo interior", cosmovisión, actitudes, valores experiencia idiosincrática, pensamiento, autoimagen y motivaciones (Romero y Gómez, 2007), que en este caso resultan vinculadas con la ingenuidad. Además, es necesario tomar en cuenta que en las prácticas de enseñanza de los profesores prevalece un enfoque subjetivo e idiosincrático con influencias pedagógicas conservadoras (Lortie, 2002).

Así pues, el profesor ingenuo tiene un particular cúmulo de actitudes, creencias, pensamientos, premisas y supuestos con respecto a la educación, la escuela, el currículo, su profesión, el alumno, la enseñanza, el aprendizaje, la evaluación y otros aspecto educativos y formativos de los cuales a menudo no está consciente; pero que le sirven de "marcos de referencia", "marcos reguladores" y de "dispositivos activadores" de su acción personal y profesional que se traducen en comportamientos concretos, en acciones docentes y en una forma particular de realizar su práctica educativa. 


\section{Pensamiento y práctica del profesor ingenuo}

A partir de estos esquemas particularmente subjetivos e idiosincráticos, el profesor ingenuo construye y proyecta su acción educativa. Dentro de esos marcos de referencia, el profesor ingenuo asume la educación y su concreción. De ahí que a menudo piense la educación desde su noción más simplista, su dimensión más sublime o su propósito más noble. A menudo asume la educación como un fenómeno ahistórico, inherentemente bueno e intrínsecamente positivo. Entiende la educación, sin ser consciente, como algo estrictamente vinculado a la transmisión sociocultural-valores social y culturalmente válidos en su sociedady en su explicación formal la reduce a su dimensión técnica-metodológica. Piensa la educación como un fenómeno o acción sin ataduras contextuales que se genera, mantiene y justifica por sí misma. Piensa la educación como el medio inalienable de superación y la entiende como "formación" aunque en su práctica privilegie la "in-formación".

Pero al profesor, desde su ingenuidad, le resulta difícil dimensionar la educación como un fenómeno contradictorio. De hecho no advierte las incoherencias, conflictos y contradicciones que plantea la educación por sí misma ni de sus consecuencias. Por un lado, reconoce y espera que la educación genere innovación, desarrollo, transformación, progreso y cambio social pero no se percata que a menudo prevalece en la práctica educativa una visión conservadora, reproductora y perpetuadora de la condición humana y de la sociedad. De igual forma, si bien se piensa en la educación como el medio para transformar, trascender, liberar y emancipar la condición humana; el hecho es que la educación ha sido utilizada -y se sigue utilizando- para manipular, oprimir, reprimir y esclavizar el desarrollo de las facultades humanas y los grupos sociales. Esas contradicciones manifiestas y latentes -escondidas y disfrazadasen los sistemas educativos, currículo y escuela pasan a menudo desapercibidos no sólo para el profesor, sino para toda la sociedad.

En adición, al profesor ingenuo le cuesta dimensionar la educación como una actividad humana condicionada por otras dimensiones igualmente humanas como la política, la económica, la social y la cultural. Difícilmente dimensiona la educación como una acción que precisamente por ser humana no solo es contradictoria e intencionada sino también imperfecta, que está sometida a un devenir histórico -que se configura y manifiesta en función de las variables en juego en un determinado momento socio-histórico-, y que está inextricablemente sujeta a los vaivenes de fuerzas externas que la instrumentalizan, la orientan y la constriñen. No puede imaginarse la educación como un instrumento que en vez de posibilitar el proyecto humano, lo reduzca, lo manipule o imposibilite, que en vez de ser usado para la liberación y la transformación del ser humano, es usado para atar, manipular y oprimir. Al profesor ingenuo le resulta difícil reconocer que la educación no es ingenua, inocente, neutral o inocua y que tampoco es ajena a los intereses e intencionalidades de personas y grupos de poder. 
El hecho es que históricamente la educación ha sido instrumentalizada para formar sujetos específicos dentro de sistemas y sociedades específicas. La forma más común de usarla es como un instrumento de integración y asimilación. Se utiliza para integrar la generación más joven dentro de la lógica del sistema y la sociedad y se acomode dentro de él. Con esta forma instrumental de entender y hacer educación precisamente se busca "amoldar", "hacer encajar", "insertar", "integrar", "adaptar" al sujeto en formación dentro de las estructuras sociales, económicas y políticas que rigen la sociedad. Así, se busca, adaptar e integrar, no cambiar. A menudo el profesor ingenuo no se da cuenta que esto lo hace él o ella como profesor a través de procesos sutiles de inculcación prolongada e intensiva dentro de la escuela. Esto sucede mientras ingenuamente se ve a sí mismo como el "agente de cambio" y cree que está formando al "nuevo" ciudadano, al "futuro" de la nación, al "nuevo" hombre y mujer que regirán los destinos de la patria o que está formando al hombre del siglo XXI. De hecho lo está haciendo, pero no en el sentido que él o ella cree.

Es más, piensa, desde una visión funcionalista, que la socialización y aculturación son las principales tareas de la educación. Cree necesario que el estudiante se adapte a un sistema de valores, códigos y significados sociales y culturales previamente establecidos para que pueda actuar e interactuar, vivir y convivir, crecer y desarrollarse dentro de dicho sistema. Entiende que es la educación la que transforma al sujeto en formación en alguien similar a las personas existentes (Delval, 1992). Por tanto, no ve nada problemático en la estructura sociocultural vigente porque de hecho no entra en su análisis crítico y no discierne sus distorsiones, desajustes e incongruencias, y si las discierne, no las vincula con la educación. Tampoco ve problemas en la transmisión y asimilación -imposiciónde un conjunto de creencias, reglas y comportamientos -que es la síntesis de su visión de educación- ya que, según su entender, estas ayudan no sólo a que una sociedad funcione, sino también a que el individuo le dé sentido a sus actuaciones y encuentre su realización personal.

El profesor ingenuo no termina de comprender que la educación, a través de las prácticas de enseñanza, conlleva necesariamente la inculcación tácita y explícita de una serie de valores -actitudes y comportamientos socialmente considerados como valiosos- muchos de ellos asociados con la disciplina, la responsabilidad, la obediencia, el respeto, el orden y aseo, las buenas costumbres y el civismo. Estos son valores necesarios, según el profesor ingenuo, para que el sujeto en formación actué de acuerde con lo que la sociedad espera de él y para que de ese modo la sociedad funcione, se desarrolle -sin sobresaltos, conflictos ni problemas, y sobre todo sin socializaciones fallidas- y se perpetúe a sí misma en esos valores. Tal como lo expresa Giroux (1997), el profesor ingenuo no percibe la intención de la educación social más allá de sus limitados resultados instruccionales más explícitos. El profesor ingenuo no logra dilucidar, para el caso, que dichos valores son los mismos que requiere la 
empresa, en un sistema capitalista, con los cuales el sujeto ha de insertarse en el mercado laboral como futuro trabajador.

La "práctica educativa" del profesor, que es en esencia una "práctica social" (Suriani, 2003), consiste en un sistema -conjunto de estructuras tanto estructuradas como estructurantes- de disposiciones duraderas que sirven como esquemas para orientar las valoraciones, percepciones y acciones de los sujetos aprendices mediante el cual dichos sujetos interiorizan lo social, y que una vez interiorizado, se constituye en el principio generador a partir del cual los sujetos generan las propias prácticas socioculturales. Esto es lo que Bourdieu (1996) denomina el habitus: un conocimiento in-corporado, hecho cuerpo, adherido a los esquemas mentales más profundos, a los dispositivos de la pre-reflexión, del "inconsciente social", con los que las personas guían la mayor parte de sus prácticas sin necesidad de racionalizarlas.

Además, el profesor ingenuo ve la educación como un fenómeno apolítico, como un fenómeno completamente desvinculado de la acción política. Bruner (1973) señala que los profesores a menudo ignoran que la enseñanza en particular, y la educación en general, tienen una naturaleza política y que esto los hace asumir la postura ficticia de neutralidad y objetividad. Raramente el profesor cae en la cuenta que a través de su práctica inculca y reproduce las intricadas relaciones de poder que sustentan el contexto de la microsociedad que constituye la escuela como réplica del marco sociopolítico más amplio de la sociedad en la que están inmersos.

Aunque no se ven a sí mismos como sujetos políticos, los profesores hacen uso de su autoridad pedagógica de forma continuada para incidir en la conformación de formas específicas de pensar y actuar de sus estudiantes. Es decir, el profesor se presenta como experto, con la investidura académica y la autoridad pedagógica-sociopolíica que le da la escuela para proponer formas especificas de pensar, sentir, valorar y hacer. Al estar en una posición de poder con respecto a los estudiantes, los induce con formas sutiles discurso y acciones pedagógicas- y formas no tan sutiles -uso de autoridad y poder: coacción, represión y castigo- a que piensen y sientan como él, a que perciban, entiendan y expliquen el mundo de acuerdo con su forma personal de entenderlo. El profesor ingenuo ve estas "formas didácticas" como inofensivas y también necesarias, y las justifica como mecanismos educativos que operan a favor de los estudiantes ya que buscan reforzar sus aprendizajes y su formación? .

Lo cierto es que, y aquí se retoma nuevamente el pensamiento de Bourdieu (1996), el profesor es un sujeto de poder. Como agente especializado dotado de autoridad, ejerce dicha autoridad a través de la inculcación que supone

1 Aunque a este punto es importante destacar que no existe un determinismo ya que los saberes escolares no son sólo repetidos por las personas, sino recreados y reinventados más allá de su canonización (Castoriadis, 1998). 
una acción pedagógica efectuada dentro de un espacio institucional -la escuela- al imponer normas arbitrarias valiéndose de técnicas disciplinarias. Además, es el profesor como sujeto político, aunque no se vea así mismo como tal, es el que además de adoctrinar y canonizar "ciertos conocimientos", toma decisiones, dispensa recompensas y sanciones, es quien legitima y deslegitima, valida e invalida, premia y castiga, controla e impone. De igual manera, aunque el profesor ingenuamente cree que la evaluación -justa, objetiva e imparcial, es el mecanismo que le sirve para "medir" y "cuantificar" los conocimientos aprendidos por el estudiante y para promover y reprobarlo, lo cierto es que inadvertida e intencionalmente, se convierte en el instrumento de poder del profesor. Es decir, usa la evaluación como un instrumento para ejercer poder más que como un instrumento pedagógico que mejora el proceso de enseñanza-aprendizaje.

Desde la ingenuidad el profesor puede llegar a creer incluso que el valor de la educación está directamente vinculado con el trabajo y el empleo. A menudo el profesor piensa que la educación solo tiene sentido si al final, ésta le sirve al estudiante para obtener un trabajo o le garantiza un empleo remunerado que le permita ganarse la vida. Cree que la razón de la educación es proporcionarle al estudiante las competencias y actitudes laborales que le permitan acceder e insertarse en el mercado laboral. Piensa que el ejercicio intelectual y los conocimientos adquiridos solo son importantes si le sirven para enfrentar los futuros retos laborales.

Pensar que el principal propósito de la educación es formar sujetos para el trabajo no solo es ingenuo sino también peligroso. Pensar que la educación debe estar en función de la empleabilidad y la empresa es una forma no sólo reducida sino también comprometida de dimensionarla. Si bien es cierto que la educación tiene el propósito explicito de la formación profesional, este no es el único propósito de la educación. Los propósitos de la educación van mucho más allá del mero desarrollo de las competencias laborales ya que la educación es es una educación para la vida -en todas sus dimensiones- y su compromiso trasciende a la humanización del hombre, implica la conquista del conocimiento y la sabiduría, la domesticación del instinto, conlleva la formación de un sujeto critico, reflexivo, sensible y propositivo tanto de su entorno natural como social; implica el desarrollo del talento y las potencialidades humanas a su máxima expresión para que encuentre su apropia realización y la de la sociedad en la que vive. La sociedad no necesita personas que vivan solo para trabajar -subordinados, manipulados y explotados por el empleador y la empresa-, sino sujetos productivos que trabajen -eficiente y eficazmente- para vivir dignamente sin postergar ni descuidar el desarrollo de toda su dimensión humana.

Desde otra arista, el profesor ingenuo concibe la educación como un proceso de formación mediatizado por una serie de procedimientos, instrumentaciones 
y tradiciones técnicas adscritas al profesor dentro de un contexto escolar. Valora el domino y aplicación de una variedad de técnicas y estrategias de enseñanza ya que estas constituyen parte esencial del ser-profesor y constituyen el bagaje que lo denota como sujeto de enseñanza. Es esta dimensión -la técnicametodológica- la que le da sentido a su labor docente. De ahí que incluso puede privilegiar el método, es decir, la forma -el cómo de la educación- por sobre el conocimiento impartido y su propósito -el qué y el para qué de la educación. Igualmente, el profesor ingenuo valora su dominio que ejerce sobre el grupo, la experticia con la que hace trabajar a sus estudiantes, el ritmo que le imprime, el control que tiene del ambiente áulico, los procesos y la conducta del estudiante. En otras palabras, la educación es una mediatización didáctica del profesor.

Considera la educación en su aspecto más puntual como algo adscrito al proceso de enseñanza y aprendizaje generalmente dentro del aula. Es decir, entiende la educación como una tarea de enseñantes y aprendices, en la cual el que enseña tiene mucho que decir, por no decir todo. Entiende que mucho del éxito o el fracaso en esta tarea depende de lo que él o ella puedan hacer ya que la mediación depende del profesor. Imposible que el profesor ingenuo conciba la educación como un proceso de formación mediatizado por otros sujetos, dentro y fuera de la escuela, o por el mundo como lo sugiere Freire; o mediatizado por los signos, las herramienta, los tiempos y el contexto sociocultural como lo propone Vygotsky.

En su auto imagen el profesor ingenuo se ve a sí mismo como un "dador de clases", aunque no siempre cae en la cuenta de ello. Se ve a sí mismo como un sujeto de y para la educación dedicado a la formación de niños y jóvenes. Se ve como sujeto de enseñanza, subjetivado en el dominio de la enseñanza (Romero, 2010). Entiende que el rol del profesor es "pararse frente" a los estudiantes para instruirlos, para impartirles o dictarles clases y, bajo esa premisa, genera toda su rutina de trabajo y su cultura docente. Siente que su tarea más sagrada consiste en enseñar, en desarrollar temas -los del plan de estudios- a través de una serie de estrategias de aprendizaje. Se ve a sí mismo como un desarrollador del currículo y siente que su lugar está en el aula, con sus alumnos.

Se ve como el sujeto adulto, modelo y formador de niños y jóvenes que sabe lo que es bueno y conviene al estudiante. Como tal, conoce la ruta, el camino y también la meta, por eso toma al estudiante de la mano y lo conduce por ese camino hacia esa meta de formación. El profesor ingenuo no se ve como el sujeto que impone la ruta, el camino y la meta, ejerciendo una práctica autocrática en un monologo permanente, dictando un discurso conjugado en modo imperativo y potencial. No se ve como sujeto de saber y poder (Foucault, 1994) ni como modelo sociocultural (Bourdieu, 1996), imbuido en dinámicas y prácticas sociales vinculadas al conocimiento que posee y transmite -no solo 
de los libros de ciencia sino de sí mismo- los saberes socioculturales a los cuales se adscribe como sujeto sociocultural.

Por otro lado, una de las dimensiones en las que el profesor demuestra más su candor y sencillez es en la noción, interpretación y aplicación del currículo. Desde la ingenuidad, el currículo es percibido como la lista de temas y objetivos educacionales a ser enseñados a los niños y muchachos en los diversos niveles. También se percibe como la organización y sistematización de las experiencias de aprendizaje o como, según Giroux (1997), la sumatoria de los cursos oficiales ofertados por una institución educativa en un programa de formación. Igualmente, se considera simplemente como la organización de lo que debe ser enseñado y aprendido, con frecuencia asumido como un proceso de toma de decisiones en el que el educador tiene poco que decir (Angulo y León, 2005).

Para el profesor, el programa de estudio, el plan de estudios o sílabo que es el currículo explicito, oficial, concreto y tangible- se convierte en la agenda educativa que dicta su quehacer docente y a la cual ha de recurrir incontablemente durante todo el proceso ya que delinea la ruta de lo que debe ser enseñado y aprendido en el curso. Ahí están las pautas de enseñanzaaprendizaje que ha de desarrollar y luego evaluar y de las que luego ha de dar cuentas al sistema educativo y la escuela a través de mecanismos de supervisión.

De ahí que el profesor asume que una de sus atribuciones primordiales está adscrita al currículo: desarrollar el programa de estudio -planeación didáctica, jornalizaciones y calendarizaciones, desagregación de objetivos, adecuaciones curriculares, mediación pedagógica y evaluación de los aprendizajes- con experticia y eficiencia. Esta tarea necesita todo su conocimiento profesional, experiencia y experticia para poder "leerlo" y aplicarlo en la forma que ahí se establece. Por todo eso se afirma que el profesor entiende el currículo desde su dimensión técnica-instrumental y así también entiende su abordaje en la práctica educativa.

Es más, asume que el currículo es una "propuesta educativa" no sólo necesaria porque guía y orienta la actividad educativa -así no tiene que crearla e reinventarla todos los días-, sino porque también conlleva las mejores intenciones y que es además inocua, sin carga ideológica, desprovista de intenciones y despojada de intereses. Igualmente piensa que el currículo no está sujeto a interpretaciones, más allá de las interpretaciones pedagógicas (Giroux, 1997) y adecuaciones curriculares necesarias, ya que los especialistas que lo generaron fueron muy cuidadosos en organizarlo, estructurarlo y sistematizarlo de acuerdo con los principios filosóficos, antropológicos y teorías educativas más actuales. Por tanto su reto como profesor no está en cuestionarlo o desafiarlo, sino en estar a la altura profesional para poder implementarlo. 
Obviamente que el currículo es mucho más complejo. Hay muchos otros aspectos sutiles pero primordiales que escapan a esta forma simplista de entender el currículo y su puesta en práctica. De ahí que, por un lado, el profesor ingenuo no alcanza a ver el currículo como la suma de todas las experiencias de vidas -compartidas y vividas- entre profesor y estudiantes dentro y fuera de la escuela que de una manera $u$ otra han incidido en la conciencia de los estudiantes y en la conformación de sus subjetividades. Es decir, no ve el currículo como el cúmulo de todas esas experiencias de vida, producto de la convivencia, relaciones, interrelaciones e interacciones entre profesor-alumno y estudiante-estudiante en un contexto sociocultural especifico que van mucho más allá de las simples prescripciones temáticas del programa y que de formas inadvertidas e impensadas han implantado valores y actitudes para la vida y que no están escritos en el currículo explícito.

De igual forma, tampoco percibe al currículo como una "negociación" o una "transacción" de tipo sociocultural, donde alumno-profesor "repiensan" y "reconstruyen", -no reproducen ni perpetuán-, su entorno sociocultural. No entiende el currículo como una construcción dialógica en construcción permanente cuyo resultado es de carácter social adscrito a un contexto y tiempo determinado. Difícilmente ve el currículo como "un instrumento" para uno u otro fin.

Por otro lado, ignora que el currículo oficial es un constructo de naturaleza política que disfraza las intenciones y relaciones de poder de los grupos hegemónicos. No puede visualizar el currículo como un instrumento ideológico usado para crear identidades y subjetividades que encajen en un sistema social, cultural, económico y político especifico. Ni lo puede visualizar como un instrumento o mecanismo de legitimación de las asimetrías y las desigualdades del orden social establecido. Igualmente, piensa que el currículo no está sujeto a interpretaciones y, por tanto, no cuestiona los axiomas que caracterizan la selección, organización y distribución del conocimiento y los estilos pedagógicos inherentes al desarrollo del currículo (Giroux, 1997).

El profesor no percibe el currículo como una imposición. No logra descubrir que el currículo es en realidad una prescripción interesada de los conocimientos científicos y socioculturales -previamente, seleccionados, parcelados, organizados, dosificados, cosificados y validados por unos pocos- que intencionalmente busca configurar en el estudiante tanto su forma de percibir el mundo como su actuación dentro de él. De hecho piensa que el conocimiento -temas y contenidos del currículo- que enseña son datos y verdades incontrovertibles, irrefutables, inalienables, objetivas, neutras, inalteradas por interpretaciones y ajenas a un sistema de valores, intereses e intenciones. Ignora que la nueva sociología despojó al currículo escolar de su inocencia hace mucho tiempo (Giroux, 1997). 
El profesor ingenuo ve en el currículo su instrumento a través del cual ha de materializar la educación, pero no se ve a sí mismo como el instrumento del currículo a través del cual el sistema materializa una forma de educación con propósitos y consecuencias que no logra comprender. El profesor ingenuo no se siente instrumentalizado por el currículo ni por el sistema educativo ya que visiona su actividad docente, en el mejor de los casos, en función y al servicio del estudiante y no ve, aunque así sea, su quehacer docente como una actividad meramente instrumental al servicio del sistema donde sus actuaciones se limitan a desarrollar un currículo prescrito desde los objetivos del Estado (Martínez, 2012).

¿Y qué decir con respecto a la escuela? Desde un marco formal, se entiende la escuela como un "centro educativo", como "el centro de formación" por excelencia de niños y jóvenes. Se visiona como el espacio físico donde estos asisten con el propósito explícito de recibir instrucción y educación. Se asume que la escuela es un centro de difusión y distribución de conocimientos donde además se desarrollan habilidades y actitudes que preparan a las personas para asumir responsablemente las tareas de la participación social en su vida adulta y les permite adaptarse a su vida social y nacional.

Desde un marco más candoroso, se ve la escuela como un "santuario" o como "el templo" donde se imparte "el pan del saber". Se percibe la escuela como un espacio desconectado -con su propia lógica y dinámicas, que se sostiene y proyecta por sí misma- y ajeno a los interjuegos sociales, económicos y políticos que suceden al otro lado de sus paredes. En palabras de Giroux (1997), la escuela parece llevar una existencia feliz más allá de los imperativos del mundo actual y sus complejidades. Es más, ha de verse como la institución encargada de la distribución equitativa del saber, por encima de las diferencias sociales, sexuales o étnicas y que por tanto contribuye a la extinción de las desigualdades y privilegios.

Desde una posición menos candorosa, la escuela es institución formadora no sólo de estudiantes, sino también de profesores. De hecho la escuela, en primera instancia, se constituye en el escenario formador de profesores donde se modela sus formas de pensar, percibir y actuar; y se constituye en el espacio donde se configuran e implementan determinadas prácticas tanto educativas como sociales (De Lella, 1999) con las cuales se genera tanto una cultura docente como una cultura institucional. Es decir, es en la escuela donde el profesor no solo aprende una forma específica de hacer las cosas con base a las fuerzas modeladoras de la institución -su práctica y cultura educativa-, sino también es el espacio donde el profesor despliega todo su poder-saber, proyecta y mediatiza su propio yo sociocultural.

Desde una posición mucho menos candorosa, yen perspectiva ya del estudiante, resulta difícil dimensionar la escuela como una "guardería". Es decir, el lugar donde llegan los padres a "depositar" a sus hijos, para que pasen el tiempo o los 
entretengan o aprendan algo, mientras ellos, como sujetos socioeconómicos, cumplen con otras funciones dentro del entramado social: el trabajo. Difícilmente se percibe la escuela de esta manera aunque se reconozca que esa es una de sus funciones latentes y el argumento se demuestre por sí mismo. Mucho más difícil resulta dimensionar la escuela como el espacio para controlar, "vigilar y castigar" o como recintos de adoctrinamiento donde se ejerce y dispensa el saber-poder en formas parecidas a la cárcel, el manicomio o el ejército, como lo afirma Foucault (1984).

Si bien se reconoce la escuela como un espacio de socialización, pero de una socialización entendida como la relación con otros sujetos de similar edad y condición o como medio para establecer relaciones de amistad y compañerismo. Se olvida a menudo que la escuela es un agente de socialización que, a través de una inculcación intensiva y prolongada, socializaimpone- los valores, creencias, visiones, reglas, disposiciones y formas de actuar propios de la sociedad en la que está inmersa. El hecho es que la escuela es la institución que la sociedad usa para sustentar valores (Ruiz y Estreval, 2006) -sociales y culturales- y formas de pensar y sentir - subjetividades- de acuerdo con los modelos de la sociedad en la cual se desenvuelve. En eso consiste la socialización escolar que el profesor ingenuo no logra reconocer porque a menudo es una enseñanza oculta -no advertida ni buscada- en las relaciones e interrelaciones dentro del aula.

Además, desde la ingenuidad resulta mucho más complicado dimensionar la escuela como institución de adaptación, asimilación, conservación, reproducción y legitimación. Pero el hecho es que a través de la escuela se impone todo el sistema cultural dominante y se subordina la cultura más débil y el sujeto es formado para adaptarse a un sistema y orden social ya existente. De igual manera, resulta difícil verla, aunque así sea, como una institución conservadora que, más que cambiar, innovar y transformar, busca preservar el patrimonio sociocultural acumulado como sociedad. Como fuerza conservadora, busca darle estabilidad y predictibilidad al sistema social existente como condición sine qua non para que la sociedad funcione y se desarrolle. Como ya lo han dejado claro varios autores (Bourdieu, 2002; Giroux, 1997, Freire, 1998), la escuela ejerce una función reproductora en el sentido que reproduce una sociedad especifica con sus valores, actitudes, formas de actuar, formas de relacionarse e interactuar. Pero también reproduce relaciones sociales de dominación, desigualdades, formas de conciencia, cargas ideológicas, valores económicos, formas y relaciones de poder. Esto es así porque la escuela, aunque no lo parezca, es una institución sociopolítica que además legitima no solo el sistema educativo sino toda la superestructura sociopolítica, cultural y económica. 


\section{Conclusión}

A manera de cierre, tratando de evitar la redundancia y retomando las palabras de Giroux (1997) se puede decir que el profesor ingenuo desconoce las complejas e intimas relaciones existentes entre la institución escolar y las instituciones económicas y políticas. A menudo ignora la relación (la naturaleza y los significados) entre la educación en las escuelas, el conocimiento escolar y el control social. No pueden esclarecer en qué modo las estructuras sociales políticas enmascaran la realidad y promueven la hegemonía ideológica. Tampoco puede dilucidar como la ordenación sociopolítica influye y constriñe los esfuerzos del individuo y colectivos para construir conocimiento y significado. No logran reconocer la relación existente entre reproducción económica y cultural.

En su forma ingenua de entender la educación y los procesos formativos, a menudo cargados de emotividad, sentimientos, fe, sinceridad y mucho romanticismo, el profesor enfatiza aquello que es explicito, que se da por cierto y por sentado. Pero falla en entender aquello que es aparente, y en desentrañar aquello que está detrás, que esta disimulado y disfrazado. Enfatiza en su práctica aquellos aspectos de forma -prácticas ritualizadas, puntualidad, presentación y aseo, "dar clases", dominio de técnicas de enseñanza, manejo del ambiente áulico y el discurso pedagógico- que constituyen el envoltorio y la parte visible de su tarea docente, pero falla en percibir las cuestiones de fondo, lo que subyace su pensamiento y práctica, las que realmente importan -la formación de conciencias.

\section{Referencias}

Angulo, L. y León, A. (2005). Perspectiva crítica de Paolo Freire y su contribución al currículo. Educere, 9(29), 159-164.

Apple, M. (1986). Ideología y curriculum. Madrid: Akal. The hidden curriculum Bourdieu, P. y Passeron, J. C. (1996). La reproducción Elementos para una teoría del sistema de enseñanza. México: Editorial Laia.

Bruner, J. (1973). The relevance of educaction. Barcelona: Paidós.

Castoriadis, C. (1998): El avance de la insignificancia. Argentina: Eudeva.

De Lella, C. (1999). Modelos y tendencias de la formación docente. Organización de Estados Iberoamericanos para la Educación y la Cultura. Consultado el 9 de julio de de 2012 en: http://www.oei.es/cayetano.htm

Delval, J. (1992). Crecer y pensar. La construcción del conocimiento en la escuela. Cuadernos de Pedagogía 3. México: Paidós

Freire, P. (1998). Politics and education. UCLA Latin American studies, 83. Los Angeles: UCLA Latin American Center Publications.

Foucault, M. (1994). Microfísica del poder. España: Planeta Agostini

Foucault, M. (1984). Vigilar y castigar. México: Siglo XXI.

Giroux, H. (1997). Los profesores como intelectuales Hacia una pedagogía crítica del aprendizaje. Madrid: Paidos. p. 63-86. 
Lerner, D. (1996). La enseñanza y el aprendizaje escolar. Alegato contra una falsa oposición" en Castorina, et al: Piaget-Vigotsky: contribuciones para replantear el debate. México: Paidós.

Lortie, D. (2002). Schoolteacher: A sociological study. Chicago: University o Chicago Press.

Martínez, N. (2012). La cara oculta del profesor. Diá-logos 10, 19-41.

Romero, O. (2010). Constitución de sujeto-profesor universitario a través de prácticas de enseñanza. Revista Virtual Universidad Católica del Norte, 31, 131-154.

Romero, J. y Gómez, A. (2007). ¿Sirven las políticas y prácticas de formación del profesorado para mejorar la educación? Una respuesta desde el análisis de la construcción social de la docencia. Archivos de Políticas Educativas, 15, 1-25.

Ruiz, E. y Estreval, L. (2006). La relación maestro-alumno en el contexto del aprendizaje. Psicología para América Latina. Recuperado de http:// psicolatina.org/Seis/ maestro.html

Suriani, B. (2003). Las prácticas de la enseñanza en contextos de cambio: características, dilemas y tensiones. Congreso Latinoamericano de Educación Superior en el siglo XXI. San Luis, Argentina. Recuperado el 9 de julio de 2012 de http://conedsup.unsl.edu.ar/Download_trabajos/ Trabajos/Eje_8_Sujetos_y_Practicas_Contextos_Crisis/Suriani_Beatriz.PDF 